Supporting Information

\title{
Bioinspired universal approaches of cavity regulation during cylinder impact processes for drag reduction in aqueous media: macro- geometry vanquishing wettability
}

Changzhuang Yao ${ }^{\ddagger 1,3}$, Yanjiao Zhou ${ }^{\ddagger 4}$, Jingming Wang $^{2 *}$, and Lei Jiang ${ }^{1,3,5}$

1. CAS Key Laboratory of Bio-inspired Materials and Interface Sciences, Technical Institute of Physics and Chemistry, Chinese Academy of Sciences, Beijing 100190, P.R. China.

2. School of Materials Science and Technology, Beijing University of Chemical Technology, Beijing 100029, P. R. China. E-mail: wangjm@buaa.edu.cn

3. School of Future Technology, University of Chinese Academy of Sciences, Beijing 101407, P. R. China.

4. Beijing E-town Experimental Primary School, Beijing 100176, P. R. China.

5. School of Chemistry, Beihang University, Beijing 100191, P. R. China. 


\section{S1. The bioinspiration of the basilisk lizards' toes towards drag reduction.}

Basilisk lizards utilized their cylindrical toes to make the air-water interface to deform and to expand downward. Consequently, an air entrained cavity formed, and the upward hydrodynamic force produced. As known, drag reduction was achieved by the stable air-entrained cavity accompanying with the downward moving objects, the outlined shape of which changed into the streamlined body. The flow separation can be suppressed, and the pressure-induced drag can be reduced. That is to say, the resistance of the sinking body at the air-water interface (Langmuir, 2008, 24, 142145) and the drag reduction (Sci. Adv. 2017, 3, e1701558; ACS Applied Materials \& Interfaces 202113 (3), 4796-4803) are two potential applications of the air-entrained cavity. To achieve the resistance of the sinking body at the air-water interface, there necessarily is no pinching-off and rupture phenomena occurred at the air-water interface. Whereas, to achieve drag reduction, the cavity must pinch off, and the lower part of cavity must move downward accompanying the objects without bursting. Although the two applications seem to be conflicted, however, they both depended on the stable air-water interface without rupture and bursting. Thus, developing a method to stabilize the air-water interface is important, both for increasing the sinking resistance and underwater drag reduction.

At present, most research focused on introducing interfacial coating to stabilize the cavity and then to achieve drag reduction especially on the spherical body, including hydrophobic coating or overheat Leidenfrost interface. (Nat. Phys. 2007, 3, 180-183; J. Fluid Mech. 2017, 823, 716-754.) However, the basilisk lizard used another method to stabilize the air-entrained cavity by utilizing their cylindrical toes, which may shed some new avenue on developing cavity stabilizing method.

\section{S2. The surface morphology of the toes of the basilisk lizard.}

The surface morphology of the basilisk lizard's foot was captured by optical camera and the three-dimensional profilometer (InfiniteFocus G5, Alicona, Austria), as shown in the Figure S1. Figure S1a is the optical photo of the lizard foot. We can see 
that the toes were covered with numerous scales. Local amplification of the toes in Figures S1b and S1c showed that the scales were arrayed densely. The length of the scales was about $1 \mathrm{~mm}$. Further amplified SEM image in Figure S1d shows that there were numerous dense holes with the diameter about 10 to $30 \mu \mathrm{m}$ (Figure S2c), the inner edges of which were composed of stacked nanosheet (Figure S2d). Thus, the toes of the basilisk lizard's foot were covered with dense scales with multiscale roughness.
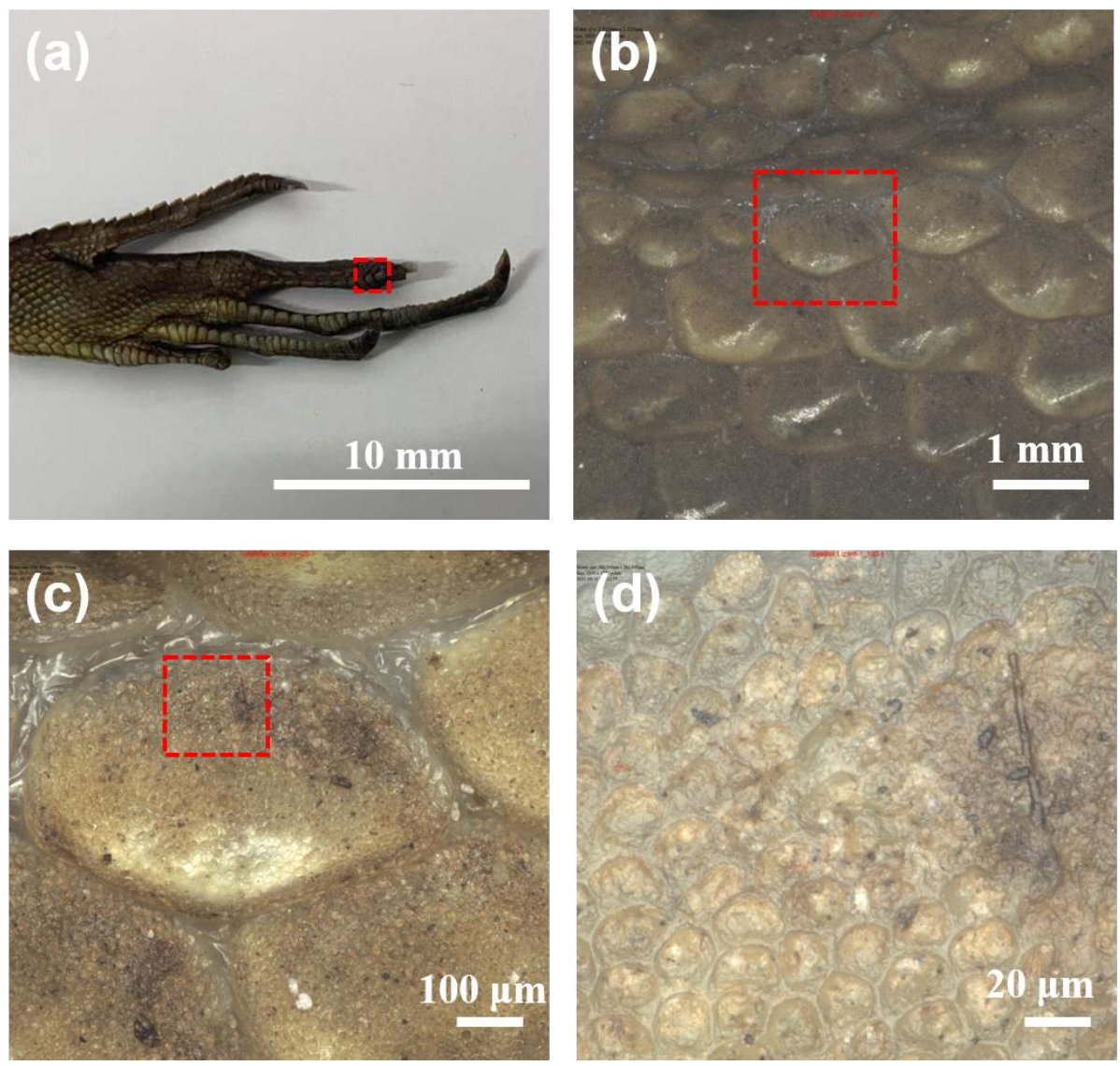

Figure S1 The surface morphology of the basilisk lizard's toe. 

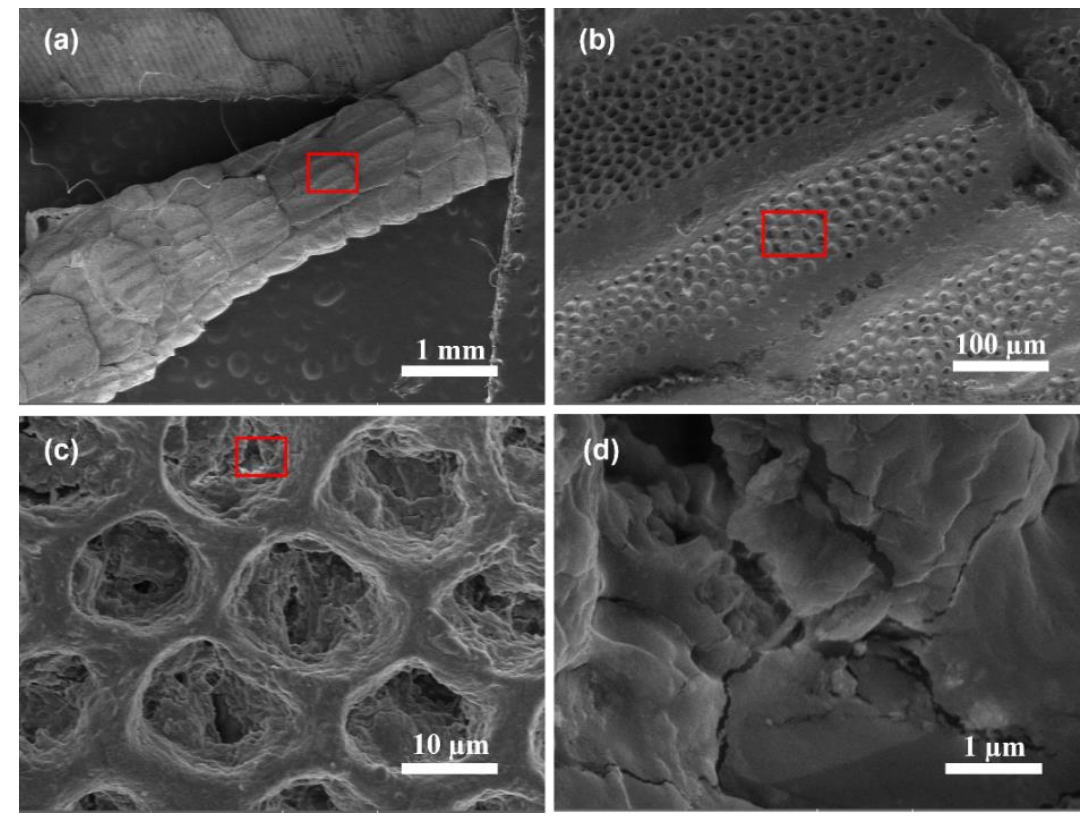

Figure S2 The SEM images of the basilisk lizard's toe.

S3. The schematic diagram of the front view, side view and vertical view of the cylinders and the amplified fillet radii

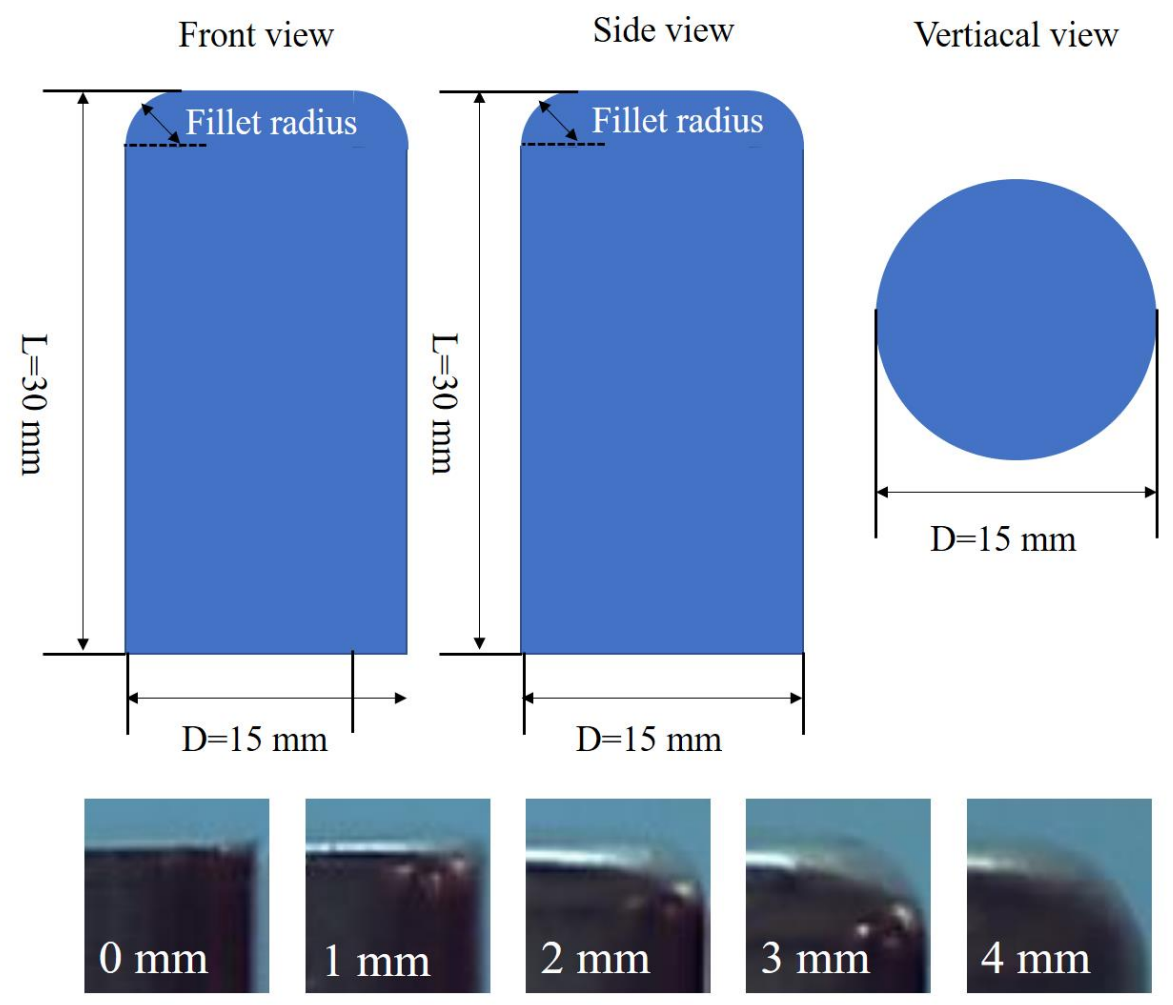

Figure S3 The schematic diagram of the front view, side view and vertical view of the cylinders with a fillet radius, and the partial amplification of cylinders with different the fillet radii of $0,1.0,2.0,3.0$, and $4.0 \mathrm{~mm}$. 
S4. Cavity formation process of cylinders with different parameters.

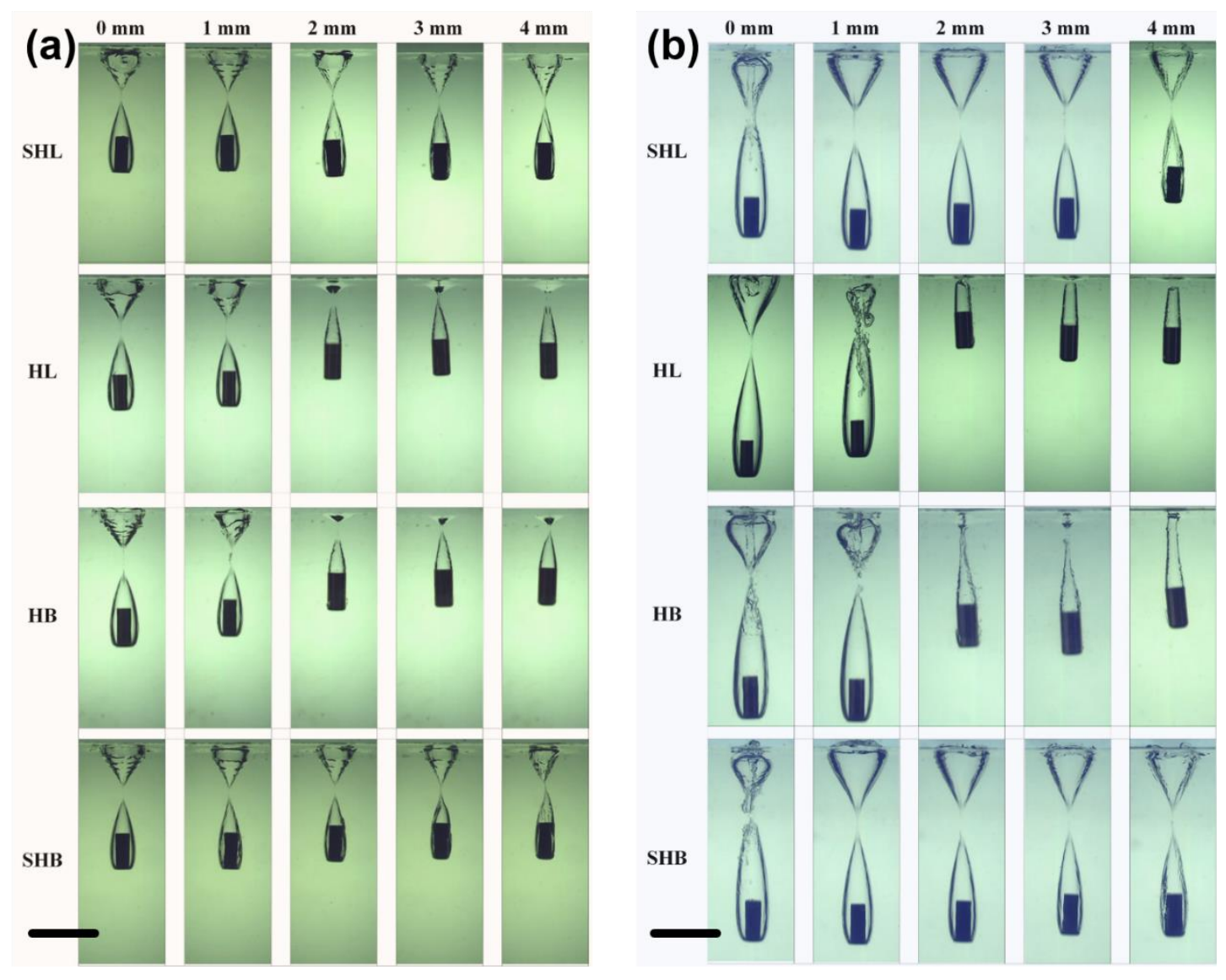

Figure S4 Selected snapshots showed the consequence of impacting the process of the cylinders with different spherical wettability (i.e., superhydrophilic, hydrophilic, hydrophobic, and superhydrophobic surfaces), different fillet radii (i.e., 0, 1.0, 2.0, 3.0 , and $4.0 \mathrm{~mm}$ ), and different releasing heights $10.0 \mathrm{~cm}$ (a) and $20.0 \mathrm{~cm}$ (b). Scale bars: $50 \mathrm{~mm}$. 


\section{S5. Average velocities measured at different depths of hydrophilic cylinders with}

different fillet radii and releasing heights

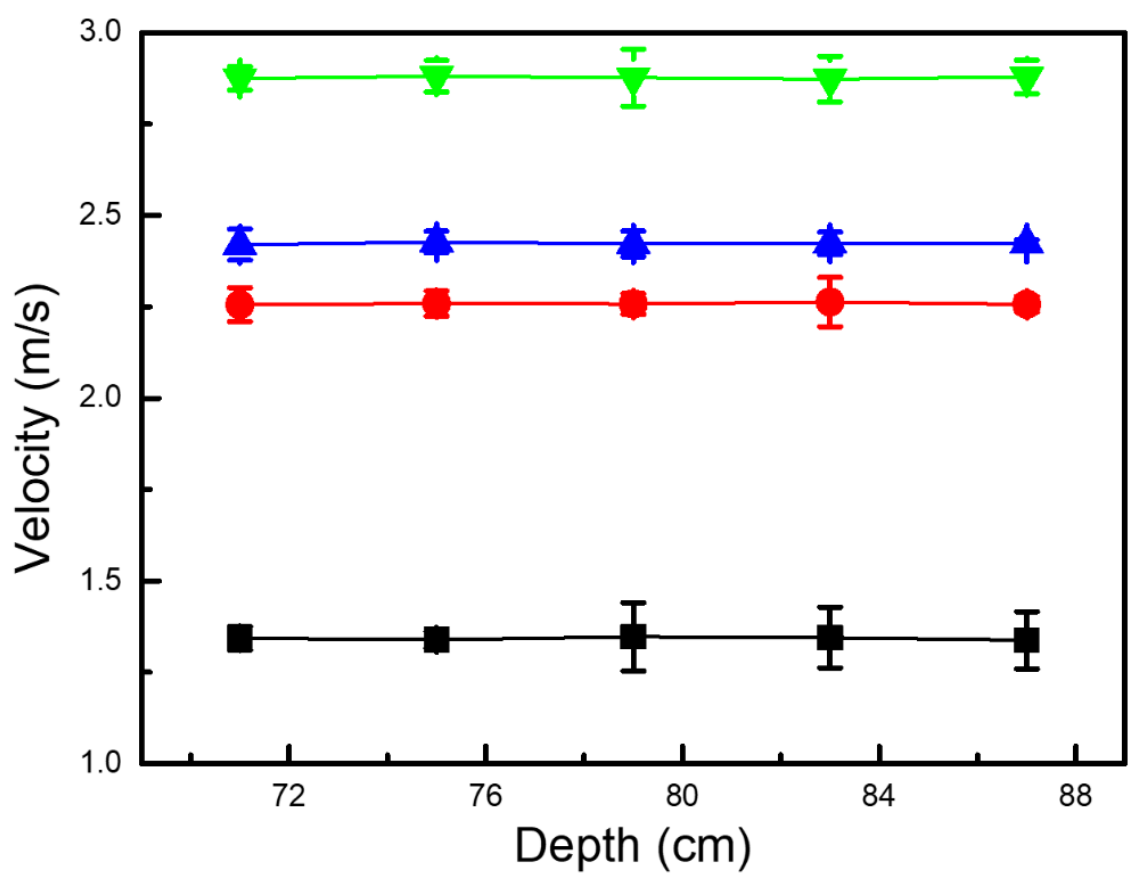

Figure S5 Average velocities measured at different depths $(71.0 \mathrm{~cm}, 75.0 \mathrm{~cm}, 79.0$ $\mathrm{cm}, 83.0 \mathrm{~cm}$, and $87.0 \mathrm{~cm}$ ) of the cylinders with different fillet radii and releasing heights (black square: fillet radius of $0 \mathrm{~mm}$, releasing height of $0 \mathrm{~cm}$; red circular: fillet radius of $0 \mathrm{~mm}$, releasing height of $40.0 \mathrm{~cm}$; blue up triangles: fillet radius of 4.0 $\mathrm{mm}$, releasing height of $0 \mathrm{~cm}$; green down triangles: fillet radius of $4.0 \mathrm{~mm}$, releasing height of $40.0 \mathrm{~cm}$ ). The average velocities were calculated by the following method: several representative frames during which the moving cylinders fell through a typical distance of $40.0 \mathrm{~cm}$ underwater were taken from the high-speed video, and from these representative frames we gathered the falling time, and then the average velocity was calculated. 
S6. Underwater uniform motion of cylinders with different parameters.

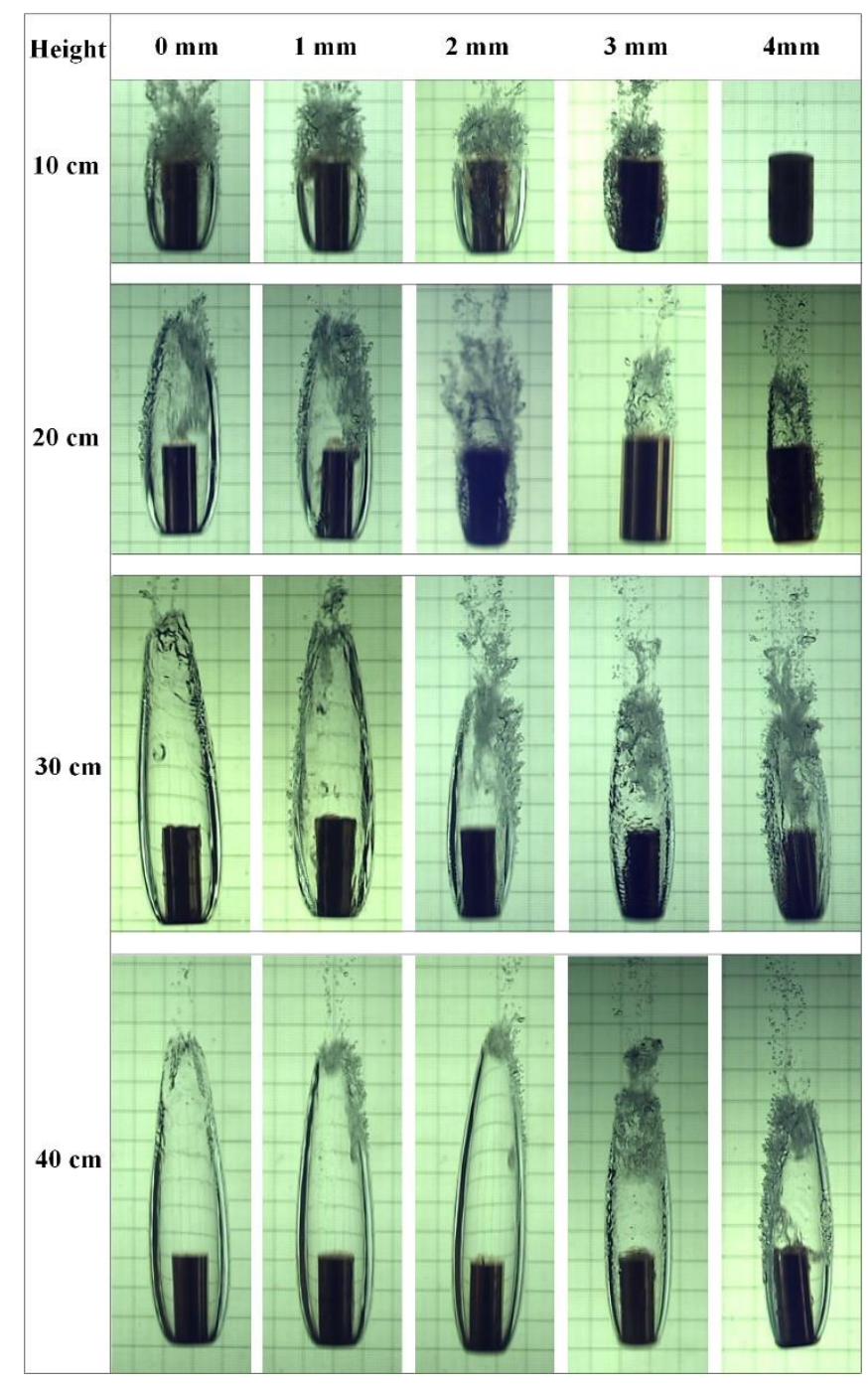

Figure S6.1 Selected snapshot showed the consequence of underwater uniform motion of the hydrophilic cylinders with different fillet radii (i.e., 0, 1.0, 2.0, 3.0, and $4.0 \mathrm{~mm}$ ) and releasing heights (i.e., $10.020 .0,30.0$, and $40.0 \mathrm{~cm}$ ). 


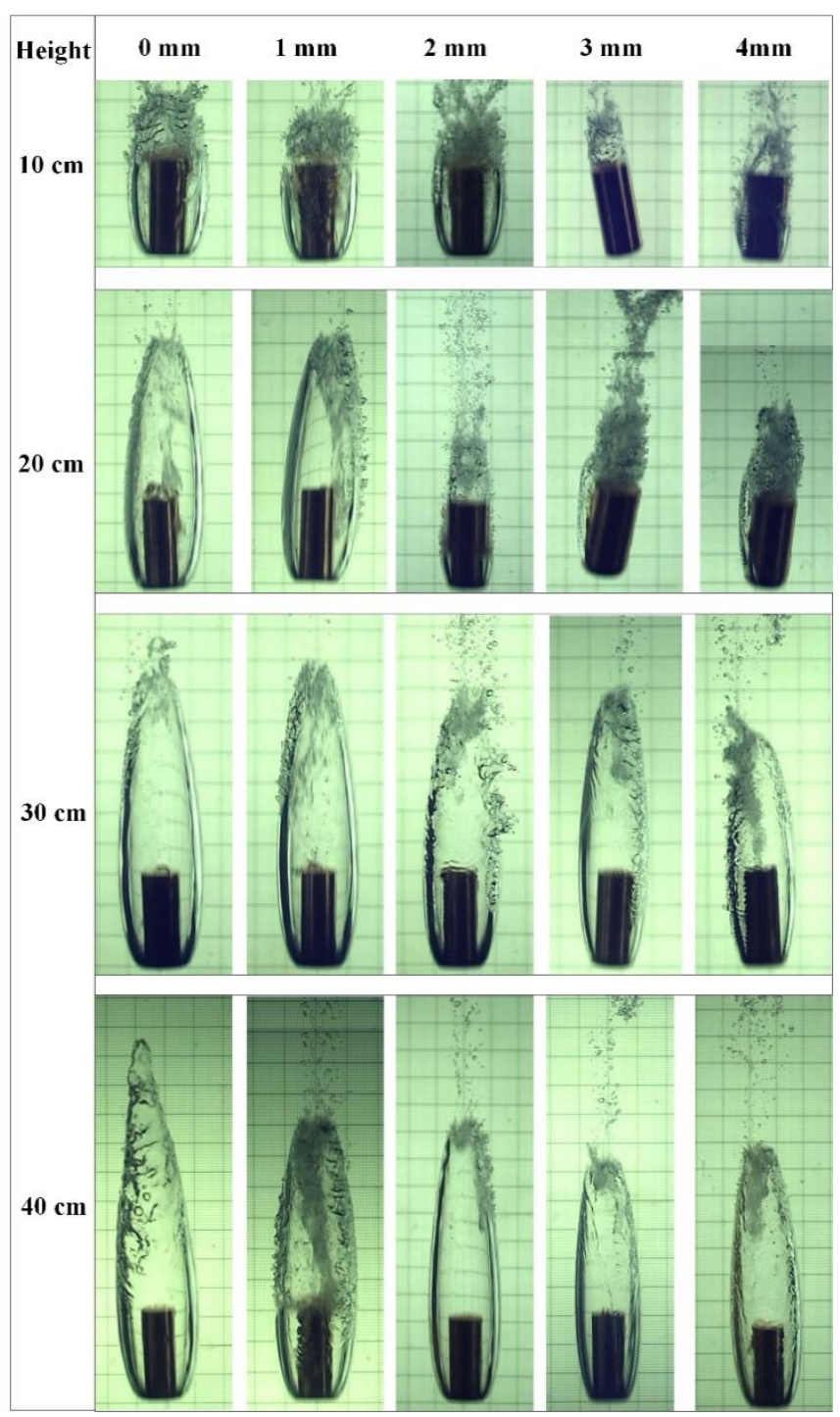

Figure 6.2 Selected snapshot showed the consequence of underwater uniform motion of the hydrophobic cylinders with different fillet radii (i.e., 0, 1.0, 2.0, 3.0, and 4.0 $\mathrm{mm}$ ) and releasing heights (i.e., 10.0, 20.0, 30.0, and $40.0 \mathrm{~cm}$ ). 


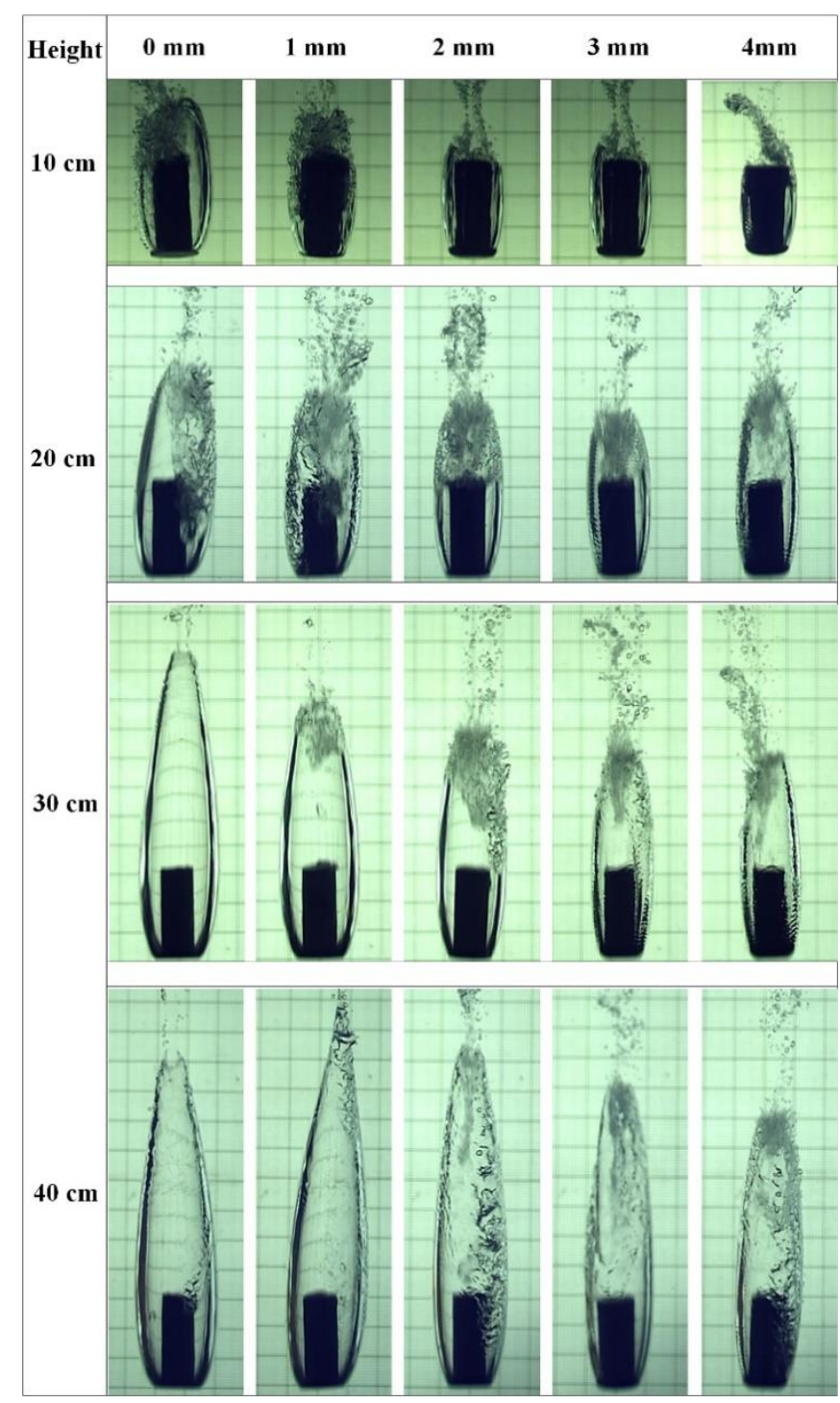

Figure S6.3 Selected snapshot showed the consequence of underwater uniform motion of the superhydrophilic cylinders with different fillet radii (i.e., 0, 1.0, 2.0, 3.0, and $4.0 \mathrm{~mm}$ ) and releasing heights (i.e., 10.0, 20.0, 30.0, and $40.0 \mathrm{~cm}$ ). 


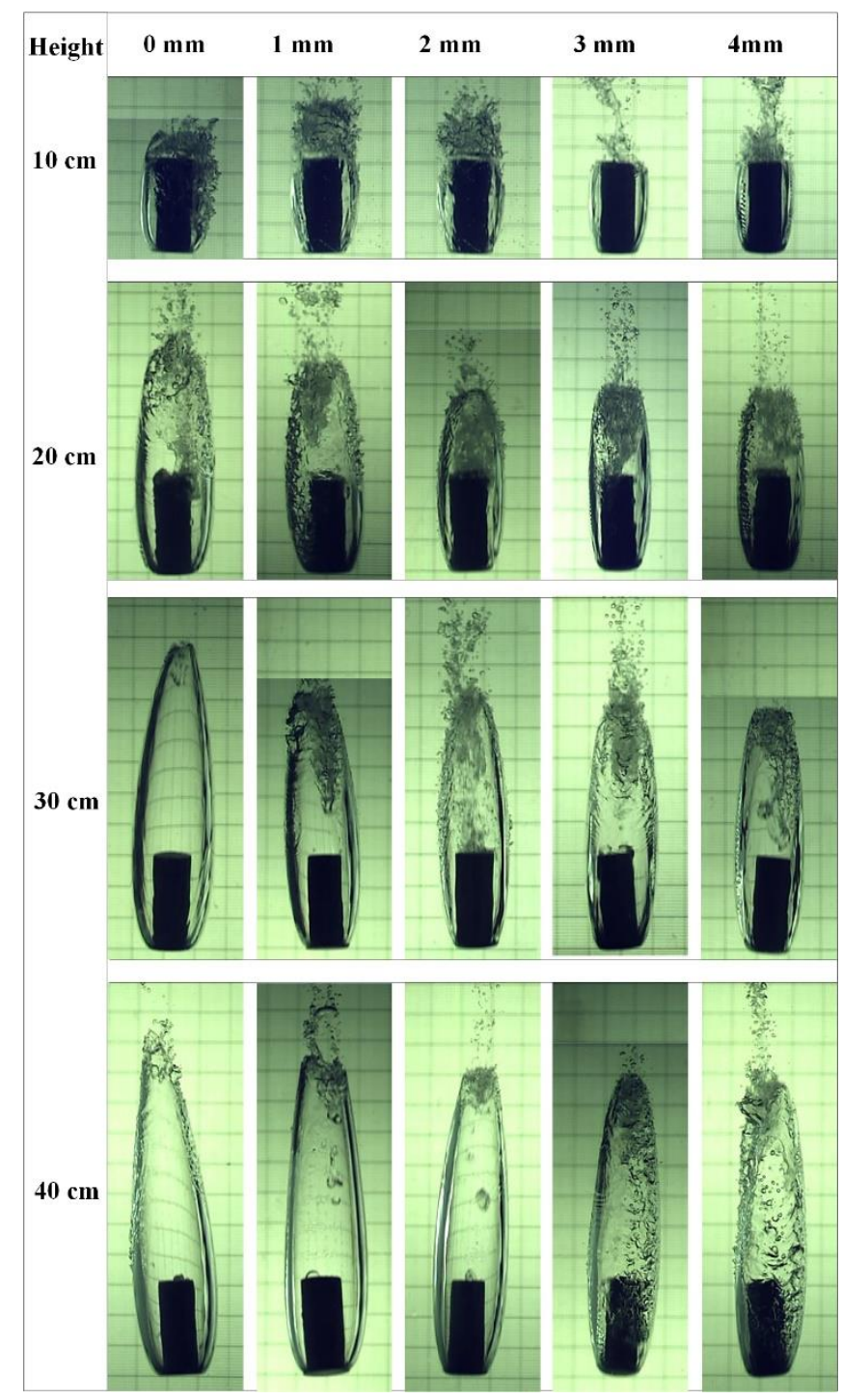

Figure S6.4 Selected snapshot showed the consequence of underwater uniform motion of the superhydrophobic cylinders with different fillet radii (i.e., 0, 1.0, 2.0, 3.0, and $4.0 \mathrm{~mm}$ ) and releasing heights (i.e., 10.0, 20.0, 30.0, and $40.0 \mathrm{~cm}$ ). 


\section{S7. The reason for the similar average velocities of the cylinders with different wettability.}

The falling cylinders with different wettabilities reached a similar terminal velocity because the surface wettability has little influenced on cavity shapes. The hydrodynamic drag force and terminal velocities of the falling cylinders were mainly determined by the outline shapes of the cavity attached on the cylinders.

Generally, the hydrodynamic drag can be divided into two parts. They are the skin friction caused by the viscosity of the fluid, and the pressure-induced drag caused by the flow separation. For the falling cylinders in our experiment, the percentage of skin friction was exceedingly small. The Re number of the falling cylinders ranged from $3.34 \times 10^{4}$ to $4.68 \times 10^{4}$. To calculate the percentage of the skin friction, we referred to some reported experimental hydrodynamic values in a published article "Journal of Fluid Mechanics 2006, 34 (4), 625-639". The values were measure from the flow past a widthways cylinder, which is different from the case in our experiment. However, to our best knowledge, there were no reported hydrodynamic value in case of the flow past a vertical cylinder. Skin friction is sensitive to the Re number and viscosity of the fluid. Flow structures near the wall of the cylinder in the cases of the referred article and our experimental were in the laminar state. Thus, we can use the hydrodynamic values from the referred article to estimate the value of skin friction. According to the referred article, the shear stress on the cylinder surface can be written as: $\tau=T \rho U^{2} \frac{1}{\sqrt{R e}}$, where $T$ is the dimensionless number, $\rho$ is the density of water, and $U$ is the velocity of the cylinder. The skin friction can be written as: $F=\tau S$, where $S$ is surface area of the cylinder. The total drag force is equal to the gravity of the cylinder, so the total drag force can be written as $D=\rho_{s} V g$, where $\rho_{s}$ and $\mathrm{V}$ are the density and volume of cylinder, respectively, $\mathrm{g}$ is the gravitational acceleration. The percentage of the skin

friction can be calculated as $\frac{F}{D}=\frac{\tau S}{\rho_{s} V \rho_{s}}=\frac{T \rho U^{2}}{\rho_{s} g \sqrt{R e}} \times \frac{S}{V}$. The value of $T$ was set as 2 (Figure 3 in the referred article). We can calculate that the percentage of skin friction was about $15 \%$. Although such an estimate is relatively rough, the result is consisted with other bluff body reported before, such as sphere and cylinders (Achenbach, 
E. Journal of Fluid Mechanics 2006, 34 (4), 625-639). Therefore, the skin friction had little influence on the total drag force. The total drag force is mainly determined by the pressure induced drag. As we known, the pressure induced drag is determined by the pressure distribution on the wall surface, which depended on the outline shape of the cavity. As shown in the Figure S6.1 to Figure S6.4, the shapes of the attached cavities on the cylinders were similar when they reached stable velocity. Consequently, the pressure-induced drags of the cylinder with different wettability were similar. Moreover, because the outline shape of the cavity attached on cylinder were similar, their drag coefficients were similar. According to the drag coefficient equation $\mathrm{C}_{\mathrm{D}}=\frac{2 \mathrm{~F}_{\mathrm{D}}}{\mathrm{U}_{S}^{2} \mathrm{\rho}_{1} \mathrm{~S}}$, the cylinders with different wettability reached the similar terminal average velocity.

\section{S8. The snapshots of underwater cavity on the cylinder surface with releasing} height of $40 \mathrm{~cm}$ at the depth region of $140.0-150.0 \mathrm{~cm}$.

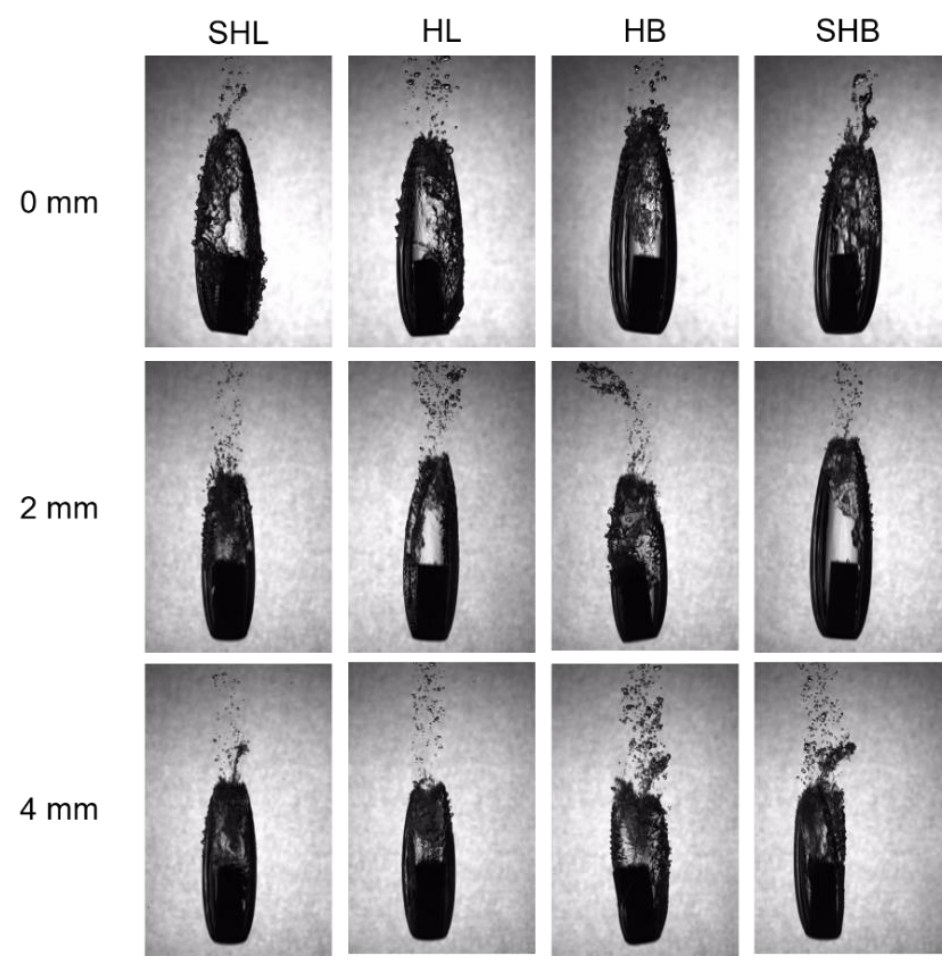

Figure S7: The snapshots of cavities on the cylinder surfaces with releasing height of $40 \mathrm{~cm}$ at the depth region of $140.0-150.0 \mathrm{~cm}$. 


\section{S9. Water impacting and developed film detachment process of cylinders with}

different parameters.

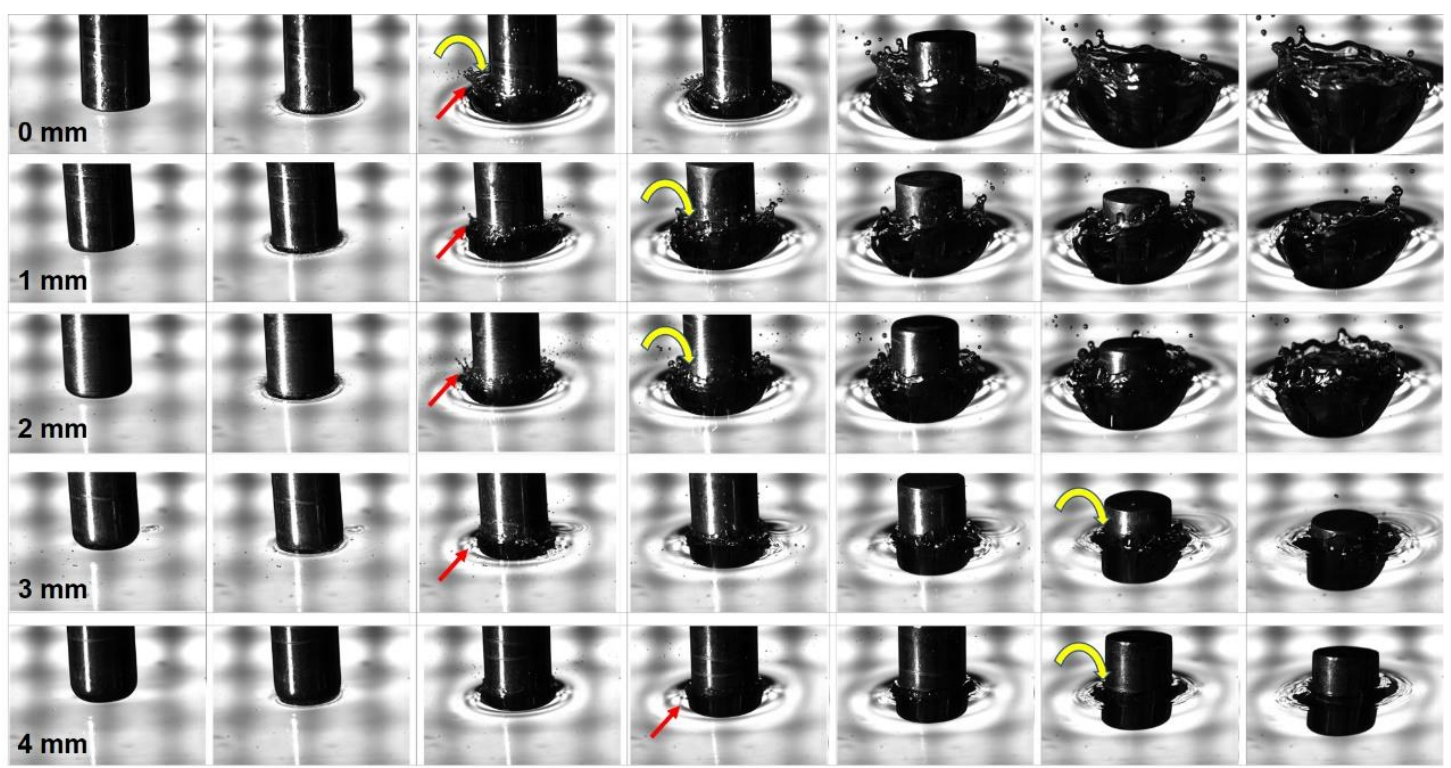

Figure S8 The detachment of the developed water film and deformation of the gasliquid interface in the water impacting the process of the cylinder with different radius diameters. The red arrows denote the detached water films, and the yellow arrows denote the air-entrainment between the cylinder surface and detached film. The first image shown was taken at the moment of impact, and subsequent images were taken at $2.0 \mathrm{~ms}$ intervals. The impact process was recorded by a high-speed video camera with a typical filming frame rate of 5,000 fps.

\section{S10. The contributions to hydrodynamic drag on the streamlined bodyand bluff body}

Generally, a streamline body is defined as the object with high slenderness ratio and sharp-edged tail, such as the waterdrop shaped objects including a submarine or torpedo. Skin friction is the key factor for the streamlined body, which is caused by fluid viscosity. A powerful way to achieve reduction of skin friction is to introduce a gas lubrication layer between the vehicle surface and its surrounding liquid to reduce skin friction. Generally, the gas lubrication layer is introduced around the vehicle surface by constructing superhydrophobic surfaces (Phys. Rev. Lett. 2011, 106(1): 014502.; Chem. 
Rev. 2015, 115(16): 8230-8293.; Phys. Rev. Lett. 2006, 97(15): 156104.) or developing supercavitation (Annu. Rev. Fluid Mech. 2010, 42: 183-203.).

In this manuscript, the rear edge of cylinder was very sharp, and the rear surface was flat and blunt. Cylinder is a bluff body. Thus, when the cylinders fall in water, the mainstream would leave the cylinder surface at the sharp edge, resulting in the flow separation and low pressure aera behind cylinder. (Journal of Fluid Mechanics, 1972, 52(2),307-320.) The falling cylinder would bear pressure-induced drag. However, when a cylinder was enfolded by the cavity, the rear part of the cylinder would change into the water drop shape, and the outlined shape of the cavity would change into the streamlined body. The flow separation can be suppressed, and the pressure-induced drag can be reduced. (Sci. Adv. 2017, 3, e1701558; ACS Applied Materials \& Interfaces 202113 (3), 4796-4803). The cavity is of vital importance for the underwater drag reduction in case of the vertical falling cylinder because the cavity can optimize the outline shape to suppress the flow separation and reduce the pressureinduced drag.

\section{S11. The hydrodynamic drag force change in the falling process}

To evaluate the hydrodynamic force change in the process of motion, we measured the average velocities of the cylinder at different depth region under water, as shown in the Figure S9. The average velocity was obtained by measuring the timescale $T$ when the cylinder went through each depth of $10 \mathrm{~cm}$, and then the average velocity

was calculated by $v=\frac{0.1}{T}$. The cylinders with fillet radius of $0 \mathrm{~mm}$ were released from the height of $0 \mathrm{~cm}$ (black square), $20.0 \mathrm{~cm}$ (red circle), and $40.0 \mathrm{~cm}$ (blue triangle) above the water surface, corresponding to the initial velocity $0 \mathrm{~m} / \mathrm{s}, 2.0 \mathrm{~m} / \mathrm{s}$, and $2.828 \mathrm{~m} / \mathrm{s}$. As shown in Figure S9, the cylinders experienced an acceleration/deceleration process at first, and then reached the terminal velocity and moved uniformly. When the initial velocity of the cylinder was smaller than the terminal velocity, the cylinder practiced an acceleration process. This result indicated that the initial hydrodynamic drag is smaller than the gravity. Because the 
hydrodynamic drag is proportion to the moving velocity, the hydrodynamic drag was gradually increased. When the hydrodynamic drag was equal to or balanced by the gravity, the cylinder would move uniformly. Similarly, when the initial velocity of the cylinder was larger than the terminal velocity, the cylinder performed a deceleration process. The initial hydrodynamic drag was larger than the gravity force, and hydrodynamic drag was gradually decreased. Moreover, the curvatures of the velocity curves were gradually decrease to 0 with the increase of depth, indicating that the acceleration speeds were gradually reduced to 0 . Our results indicates that the falling cylinders went through steady acceleration/deceleration processes.

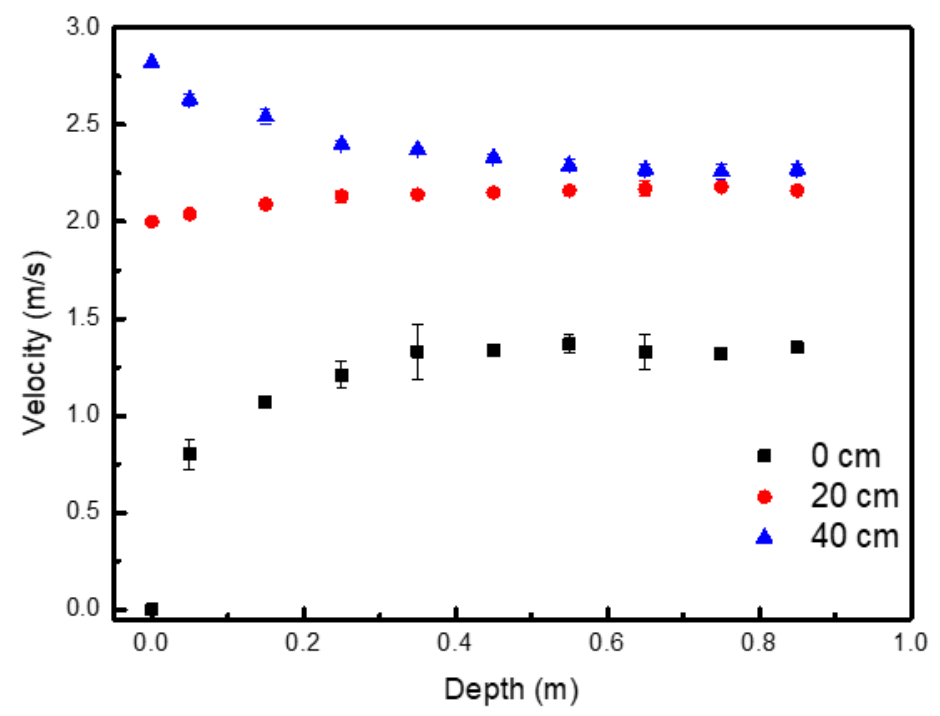

Figure S9 The measured average velocities of the falling cylinders at different depth region under water.

\section{S12 The validity of the comparison with the cylinders from a height of $0 \mathrm{~cm}$}

In our experiments, there are two factors can influence the terminal velocities of the falling cylinders. One is the releasing height; the other is the fillet radius. When the cylinders were released from the height of $0 \mathrm{~cm}$, no cavities attached to the underwater cylinders. However, when the cylinders were released from a height larger than $0 \mathrm{~cm}$, cavities formed and enfolded the cylinder surface to change their outline 
shapes. At the same time, the terminal velocities were increased, resulting from the drag reduction effect of attached cavity.

The velocities plotted in Figure 3 were the terminal velocities of the cylinders. The cylinders moved uniformly in the force balanced states. In that case, the gravity was balanced by the upward hydrodynamic drag, $G=F_{\text {drag }}=\frac{1}{2} C_{D} S \rho v^{2}$, where $C_{D}$ is the drag coefficient, $S$ is the maximal crossing area, $\rho$ is the density of water, and $v$ is the terminal velocity of the cylinder. The value of $C_{D}$ is only determined by the outline shapes of the cavity, $S$ and $\rho$ are the intrinsic characters of cavity and water, respectively. Thus, the terminal velocity showed no dependence on the initial velocity, and only determined by the outline shapes of the cavity attached cylinders. This conclusion was demonstrated by the cases of the impacting spheres in our previous paper. (ACS Applied Materials \& Interfaces 202113 (3), 4796-4803). If a sphere impacted the water without any cavity being produced (the outline shapes did not change), the terminal velocities of the sphere exhibited no dependence on the releasing height (Table S5 to S8 in the paper ACS Applied Materials \& Interfaces 202113 (3), 4796-4803). For the spheres with cavity attached, some results of the papers from Thoroddsen's group also demonstrated that the terminal velocity depended on the cavity shapes, instead of the initial velocity. (Figure 13 in the paper “J. Fluid Mech. 2017, 823, 716-754”; Figure S5 in the paper "Sci. Adv. 2017, 3, e1701558”). In our experiment, the cavity can be induced for all the cylinders with different wettability at the releasing height larger than $0 \mathrm{~cm}$. Thus, although the cylinders were released from different heights impacting the water surface with different initial velocities, their terminal velocities were only determined by the outline shapes of the cavity, regardless of their initial velocity. Therefore, we considered that the comparisons with the cylinders from a height of $0 \mathrm{~cm}$ are valid.

S13. The calculated $\mathrm{CD}_{\mathrm{D}-\mathrm{TDC}}$ of the cylinders with different wettability 
Table S1. The calculated $C_{D-T D C}$ of the hydrophobic cylinders with different releasing heights and fillet radii.

\begin{tabular}{cccccc}
\hline Reight $(\mathrm{cm})$ & 0 & 1.0 & 2.0 & 3.0 & 4.0 \\
\hline 0 & 1.307 & 1.201 & 1.073 & 1.070 & 0.969 \\
\hline 10.0 & 0.567 & 0.474 & 0.365 & 0.361 & 0.348 \\
\hline 20.0 & 0.539 & 0.459 & 0.341 & 0.365 & 0.321 \\
\hline 30.0 & 0.516 & 0.433 & 0.337 & 0.316 & 0.295 \\
\hline 40.0 & 0.497 & 0.413 & 0.330 & 0.303 & 0.279 \\
\hline
\end{tabular}

Table S2. The calculated $\mathrm{C}_{\mathrm{D}-\mathrm{TDC}}$ of the superhydrophilic cylinders with different releasing heights and fillet radii.

\begin{tabular}{cccccc}
\hline Radius $(\mathrm{mm})$ & 0 & 1.0 & 2.0 & 3.0 & 4.0 \\
\hline 10.0 & 1.319 & 1.209 & 1.121 & 1.058 & 0.961 \\
\hline 20.0 & 0.524 & 0.508 & 0.424 & 0.367 & 0.327 \\
\hline 30.0 & 0.520 & 0.456 & 0.401 & 0.369 & 0.334 \\
\hline 40.0 & 0.502 & 0.435 & 0.346 & 0.301 & 0.293 \\
\hline & 0.474 & 0.411 & 0.322 & 0.291 & 0.259 \\
\hline
\end{tabular}


Table S3. The calculated $\mathrm{C}_{D-T D C}$ of the superhydrophobic cylinders with different releasing heights and fillet radii.

\begin{tabular}{cccccc}
\hline 0 & 0 & 1.0 & 2.0 & 3.0 & 4.0 \\
\hline 10.0 & 0.550 & 0.486 & 0.396 & 0.355 & 0.326 \\
\hline 20.0 & 0.522 & 0.443 & 0.352 & 0.346 & 0.319 \\
\hline 30.0 & 0.508 & 0.443 & 0.352 & 0.298 & 0.312 \\
\hline 40.0 & 0.487 & 0.413 & 0.326 & 0.276 & 0.265 \\
\hline
\end{tabular}

S14. The imapcting processes and drag reduction effect of the cylinder ending with half-sphere (fillet radius $7.5 \mathrm{~mm}$ ).

We prepared the cylinders ending with half-sphere (corresponding to the fillet radius of $7.5 \mathrm{~mm}$ ), which were modified with different microstructures and chemical components by using the same methods of the truncated cylinders (Figure S10). The dynamic impacting behaviors of the cylinders with half-sphere ends were investigated by the high-speed camera (ispeed 713, IX, The U.K.) with frame rate of $2000 \mathrm{ftp} / \mathrm{s}$. The successive snapshots of the water impacting processes of hydrophilic, hydrophobic, superhydrophilic and superhydrophobic cylinders with half-sphere ends were plotted in Figure S11. 


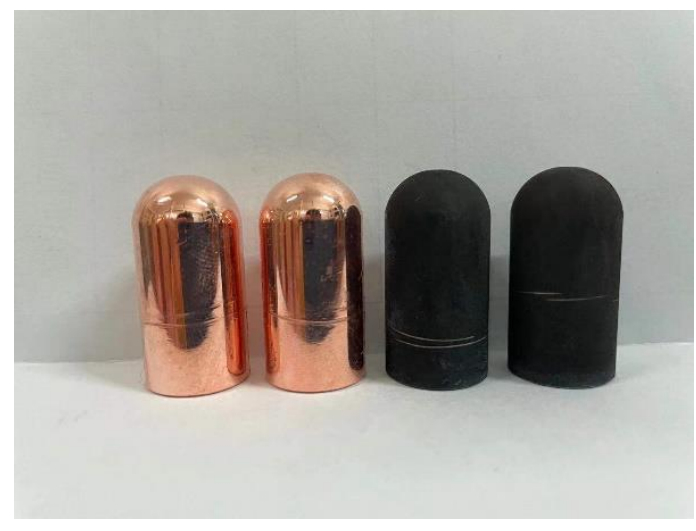

Figure S10 The prepared half-sphere ending cylinders (corresponding to the fillet radius $7.5 \mathrm{~mm}$ ) with different wettability. From left to right: hydrophilic, hydrophobic, superhydrophilic, and superhydrophobic cylinders.

At the initial stage of impacting, a thin film will develop and tend to wet the surface of impacting object (Nat. Phys. 2007, 3, 180-183). As for the hydrophilic, hydrophobic and superhydrophlic cylinder surfaces, the water films wetted the cylinder side surfaces without detachment at $10 \mathrm{~ms}$. Thus, no air was entrained at the initial impacting stage, and the side surfaces were fully wetted. After these cylinders immersing under water, the contact lines pinned at the sharp edges. Finally, the cavities pinched off near the cylinder surfaces, i.e., "surface seals" occurred, resulting in small cavities attached to the rear parts of the cylinders. As for the superhydrophobic cylinder surface, a large splash was observed above the water surface at the initial stage, resulting in the air entrapment and cavity formation. The cavity pinched off at the middle part and relative deep location, i.e., the "deep seal" occurred. Thus, the cavity fully enfolded the side surface of the superhydrophobic cylinder. The dynamic wetting behavior of the water film on half-spherical surface was in accordance with the case of the impacting spheres (ACS Applied Materials \& Interfaces 202113 (3), 4796-4803). The initial wetting behavior of the thin water film on the cylinder with half-sphere ends was sensitive to the surface wettability, and further influenced the development processes and shape of subsequent cavities. 

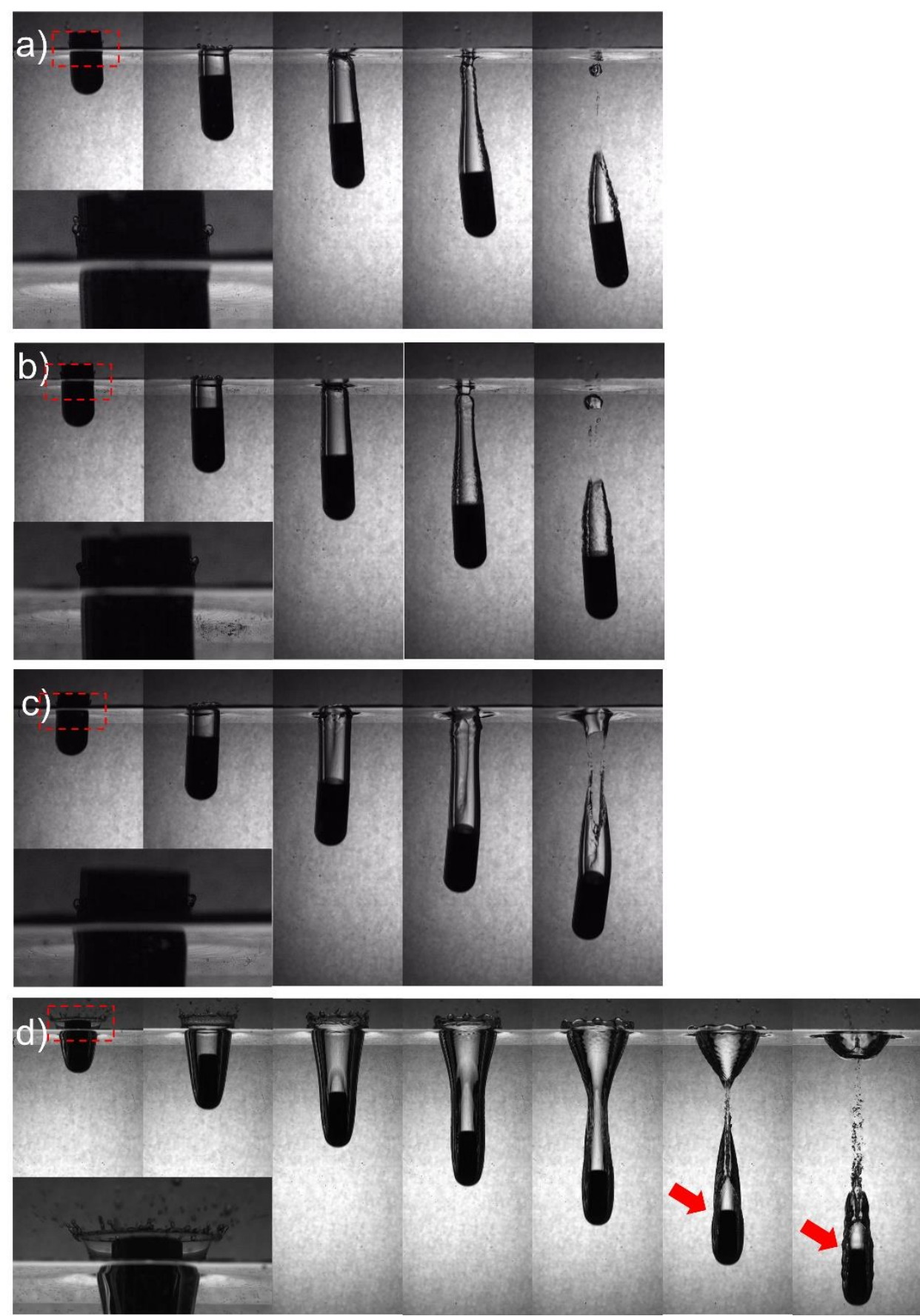

Figure S11 Successive snapshots of the water impacting processes of (a) hydrophilic, (b) hydrophobic, (c) superhydrophilic and (d) superhydrophobic cylinders with halfsphere ends. Every first snapshot was taken at $10 \mathrm{~ms}$ after impacting, and subsequent snapshots were taken at each interval of $10 \mathrm{~ms}$.

After investigating the dynamic impacting behaviors of the non-superhydrophobic and superhydrophobic cylinders with half sphere ends at different heights, we found they were quite different (Figure S12). The cavities on the non-superhydrophobic 
cylinders were observed only attached to the rear of cavity, and the contact line were pinned at the rear sharp edge, while the cavity on the fully enfolded the cylinder surface. The volume of entrapped air on superhydrophobic cylinders was much larger than that on non-superhydrophobic cylinders.

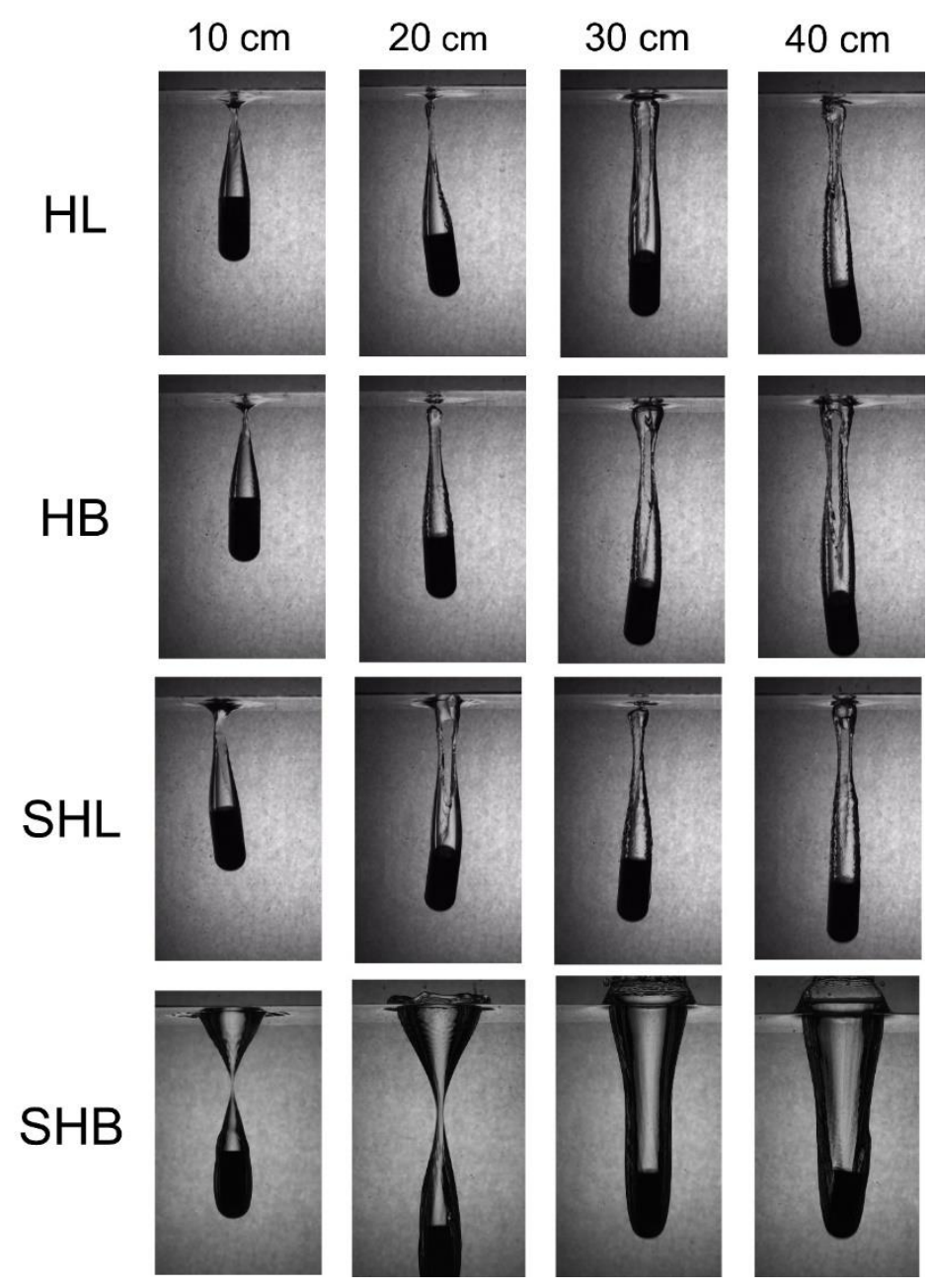

Figure S12 Selected snapshots showed the consequence of the impacting of the halfsphere ending cylinders with different wettability (HL: hydrophilic surfaces, HB: hydrophobic surfaces, SHL: superhydrophilic surfaces, and SHB: superhydrophobic surfaces) and different releasing heights (i.e., 10.0, 20.0, 30, and $40 \mathrm{~cm}$ ). 

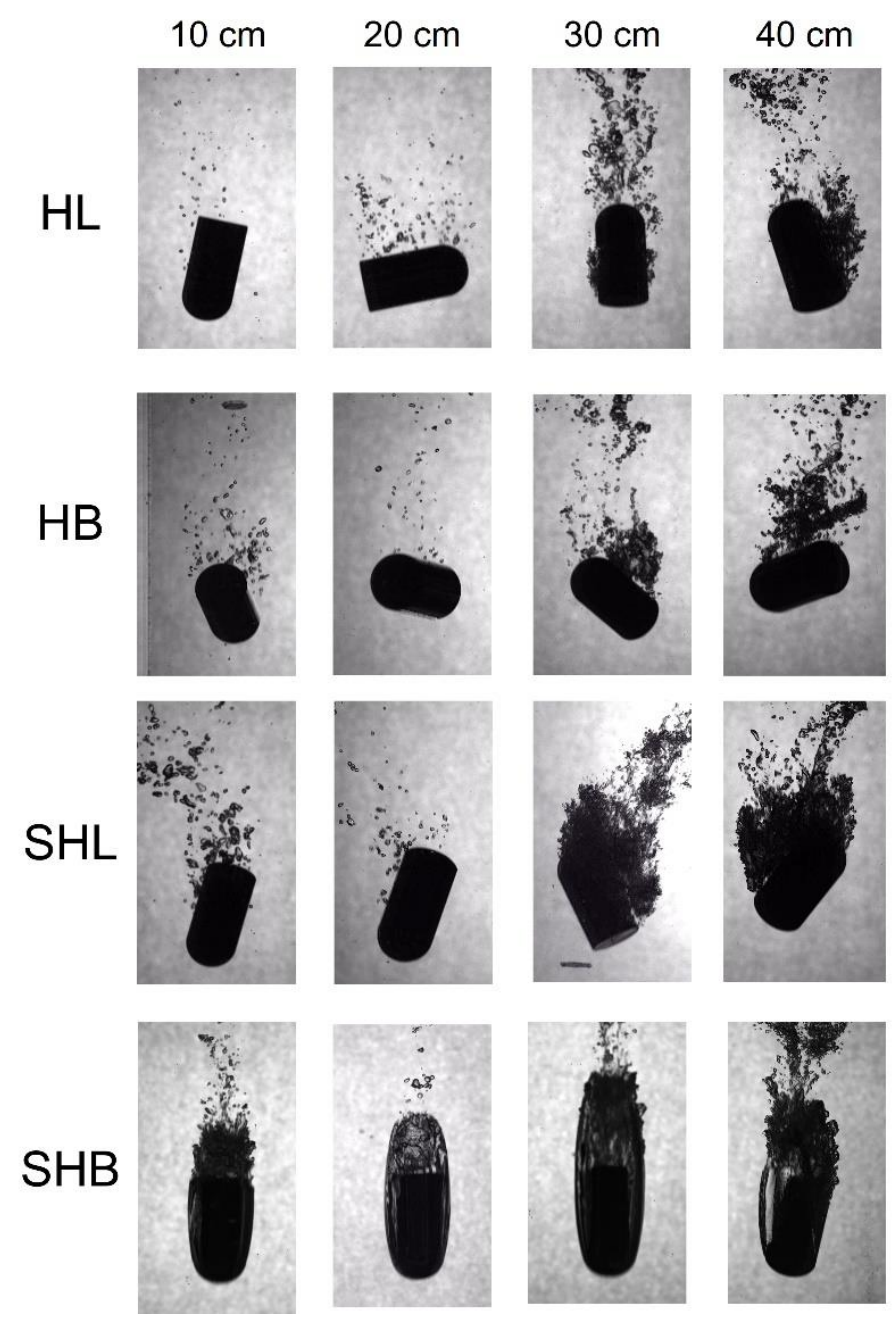

Figure S13 Selected snapshots of half-sphere ending cylinders with different spherical wettability (HL: hydrophilic surfaces, HB: hydrophobic surfaces, SHL: superhydrophilic surfaces, and SHB: superhydrophobic surfaces) and different releasing heights (i.e, 10.0, 20.0, 30.0, and $40.0 \mathrm{~cm}$ ) at depth region 70.0 to $90.0 \mathrm{~cm}$.

We further captured the snapshots of the falling cylinders in the depth region 70 to $90 \mathrm{~cm}$ under water, as shown in the Figure S13. The non-superhydrophobic rotated with varied angles, and the cavity detached from them, resulting in numerous small bubbles being released behind the cylinders. The blunt spherical head of the cylinder brought the flow separation. The unsteady force induced by the flow separation made the falling cylinders rotate and be unstable. The rotating behavior can also be found as for the bare cylinder without cavity (Figure S14a). However, for the superhydrophobic cylinder, the attached cavity kept attaching on their surface. There 
were numerous wrinkles observed on the cavity surface. Small bubbles were continuously shed from the tail of the cavity (Figure S14b). We considered that the bubble shedding behavior originated from the pinching off behavior of the cavity, as shown in the Figure 2a. Before the cavity pinched off, the middle part of the cavity started to contract. As marked by the red arrow, the contraction was impeded by the rare sharp edge of the cylinder. Consequently, the cavity became unstable and bubbles shedding from the tail. Furthermore, the superhydrophobic cylinder enfolded by the cavity can keep vertical falling without rotating. The flow separation was suppressed by the cavity because the outline shape of the cylinder was more streamlined.
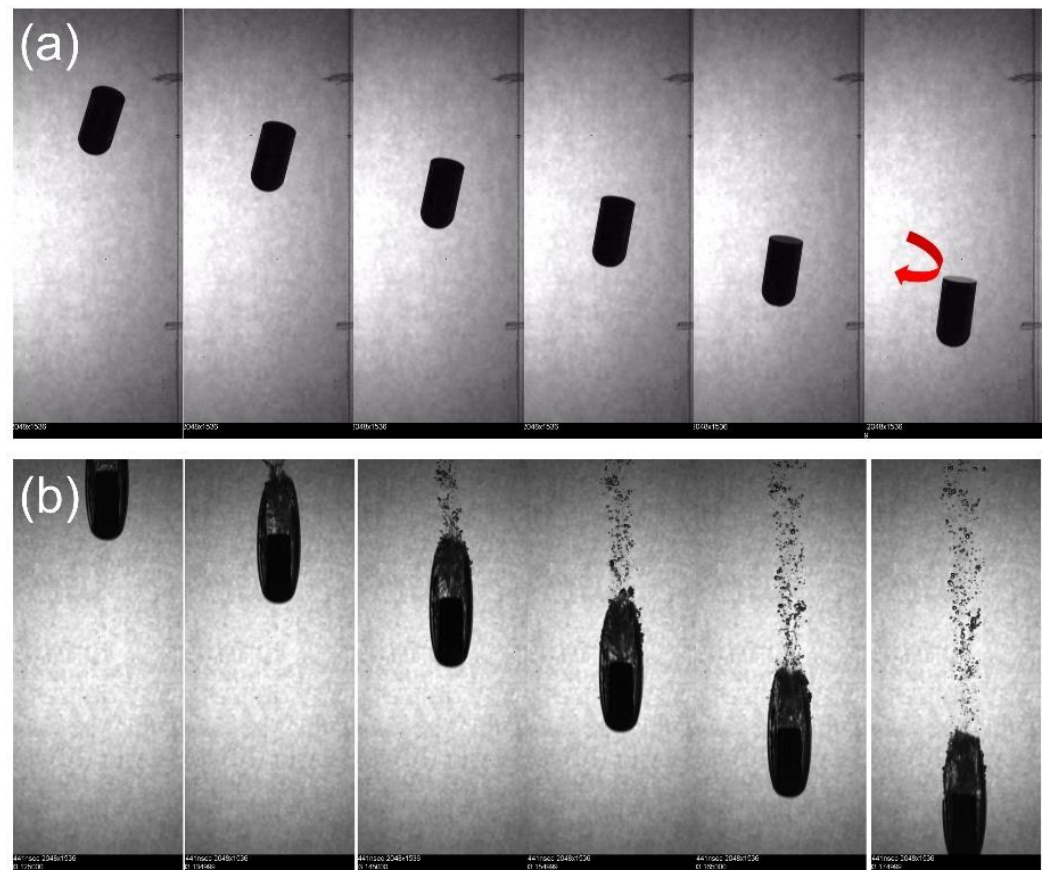

Figure S14 Successive snapshots of the underwater cylinders with half-sphere ends at depth region 70.0 to $90.0 \mathrm{~cm}$ : (a) the hydrophilic cylinder released from the height of $0 \mathrm{~cm}$ and (b) the superhydrophobic cylinder released from the height of $20 \mathrm{~cm}$.

Finally, we measure the velocities of the cylinders with half-sphere ends, as shown in Figure $\mathbf{S 1 5}$ at depth region 70.0 to $90.0 \mathrm{~cm}$. The terminal velocities of nonsuperhydrophobic cylinder fluctuated slightly with the increase of releasing height, because the cavity detached from the cylinder. The terminal velocities of the 
superhydrophobic cylinders increased with the releasing heights, indicating that the drag forces were significantly decreased by the attached cavities. We calculated the $\mathrm{C}_{\mathrm{D}-\mathrm{TDC}}$ of the superhydrophobic cylinders with different releasing heights. The $\mathrm{C}_{\mathrm{D}-\mathrm{TDC}}$ was $0.878,0.393,0.314,0.267$, and 0.248 at the releasing height of $0 \mathrm{~cm}, 10.0 \mathrm{~cm}$, $20.0 \mathrm{~cm}, 30.0 \mathrm{~cm}$, and $40.0 \mathrm{~cm}$, respectively. Thus, if increasing the fillet radii of the superhydrophobic cylinders to $7.5 \mathrm{~mm}$ can reduce the total drag force to $71.7 \%$, which was higher than the value of the cylinders with the fillet radius of $4.0 \mathrm{~mm}$.

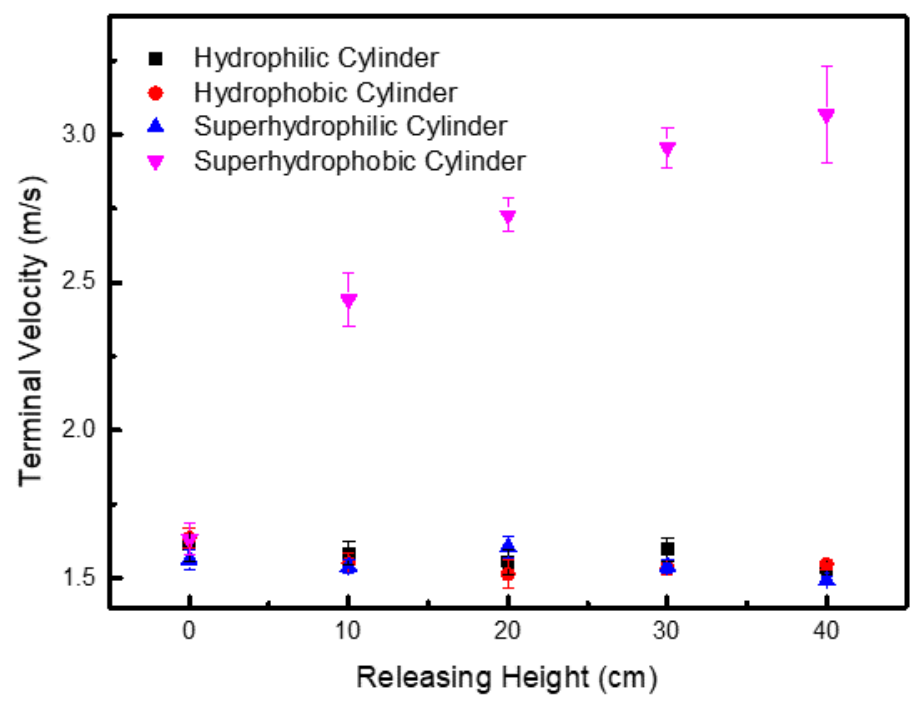

Figure S15 The measured velocities of the half-sphere ending cylinders with different spherical wettability and different releasing heights (i.e., 10.0, 20.0, 30.0, and 40.0 $\mathrm{cm})$ at depth region 70.0 to $90.0 \mathrm{~cm}$.

\section{S15. The comparation on hydrodynamic drag reduction effect of the cavity}

The drag coefficient $C_{D}$ of the spheres is calculated by using the following equation.

$$
\mathrm{C}_{\mathrm{D}}=\frac{2 \mathrm{~F}_{\mathrm{HD}}}{\mathrm{U}^{2} \rho_{1} \mathrm{~S}}
$$

where $F_{H D}$ and $U$ are the hydrodynamic drag force and measured terminal velocity of the spheres, respectively, $\rho_{l}$ is the density of the water, and $S$ is the selected crossing section area. When the sphere moved uniformly and reached the terminal velocity, the resultant force of the $F_{H D}$ and buoyancy force was balanced by the gravity force. 
To compare the drag reduction effect of the cavity attached on the spheres and cylinders, we calculated the drag coefficient $C_{D}$ of cylinders with different wettability and fillet radii at different releasing heights, as shown in the Tables S4 to S7. We found that the drag coefficient of the cylinders was significantly reduced, indicating that the hydrodynamic drag significantly reduced by the attached cavity. For example, in case of the hydrophilic cylinders with fillet radii of $0 \mathrm{~mm}, 1 \mathrm{~mm}, 2 \mathrm{~mm}, 3 \mathrm{~mm}$, and $4 \mathrm{~mm}$, when the releasing height increased from 0 to $40 \mathrm{~cm}$, the $C_{D}$ decreased from $1.170,1.071,0.943,0.894$ and 0.819 to $0.099,0.104,0.118,0.129$ and 0.138 , respectively. Consquently, the hydrodynamic drag reduction efficiency were $91.5 \%$, $90.3 \%, 87.58 \%, 85.6 \%$, and $83.2 \%$. Thus, the cavities attached on the cylinders can reduce the hydrodynamic drag to a range of $80 \%$ $90 \%$, which was closed to hydrodynamic drag reduction on the superhydrophobic spheres (ACS Applied Materials \& Interfaces 2021, 13, 4796-4803).

Table S4. The calculated $\mathrm{C}_{\mathrm{D}}$ of the hydrophilic cylinders with different releasing heights and fillet radii.

\begin{tabular}{cccccc}
\hline Radius $(\mathrm{mm})$ & 0 & 1.0 & 2.0 & 3.0 & 4.0 \\
\hline 0 & 1.170 & 1.071 & 0.943 & 0.894 & 0.819 \\
\hline 10.0 & 0.457 & 0.357 & 0.343 & 0.332 & 0.282 \\
\hline 20.0 & 0.262 & 0.236 & 0.237 & 0.225 & 0.235 \\
\hline 30.0 & 0.114 & 0.094 & 0.140 & 0.167 & 0.176 \\
\hline 40.0 & 0.099 & 0.104 & 0.118 & 0.129 & 0.138 \\
\hline
\end{tabular}

Table S5. The calculated $C_{D}$ of the hydrophobic cylinders with different releasing heights and fillet radii. 


\begin{tabular}{cccccc}
\hline Reight $(\mathrm{cm})$ & 0 & 1.0 & 2.0 & 3.0 & 4.0 \\
\hline 0 & 1.139 & 1.047 & 0.935 & 0.932 & 0.844 \\
\hline 10.0 & 0.457 & 0.377 & 0.307 & 0.292 & 0.303 \\
\hline 20.0 & 0.250 & 0.246 & 0.168 & 0.228 & 0.219 \\
\hline 30.0 & 0.120 & 0.096 & 0.129 & 0.145 & 0.165 \\
\hline 40.0 & 0.094 & 0.117 & 0.147 & 0.146 & 0.139 \\
\hline
\end{tabular}

Table S6. The calculated $C_{D}$ of the superhydrophilic cylinders with different releasing heights and fillet radii.

\begin{tabular}{cccccc}
\hline Radius (mm) & 0 & 1.0 & 2.0 & 3.0 & 4.0 \\
\hline 0 & 1.151 & 1.054 & 0.977 & 0.922 & 0.838 \\
\hline 10.0 & 0.438 & 0.407 & 0.360 & 0.284 & 0.268 \\
\hline 30.0 & 0.252 & 0.269 & 0.256 & 0.253 & 0.209 \\
\hline 40.0 & 0.119 & 0.163 & 0.169 & 0.172 & 0.182 \\
\hline
\end{tabular}

Table S7. The calculated $C_{D}$ of the superhydrophobic cylinders with different releasing heights and fillet radii. 


\begin{tabular}{cccccc}
\hline Radius $(\mathrm{mm})$ & 0 & 1.0 & 2.0 & 3.0 & 4.0 \\
\hline 10.0 & 1.139 & 1.047 & 0.935 & 0.933 & 0.844 \\
\hline 20.0 & 0.421 & 0.391 & 0.329 & 0.297 & 0.275 \\
\hline 30.0 & 0.245 & 0.211 & 0.181 & 0.184 & 0.186 \\
\hline 40.0 & 0.115 & 0.191 & 0.180 & 0.159 & 0.178 \\
\hline & 0.063 & 0.065 & 0.106 & 0.114 & 0.107 \\
\hline
\end{tabular}

If we consider the increasement of the terminal velocity, we found that cylinders with fillet radius of $4.0 \mathrm{~mm}$ showed a higher rising rate. For example, when the releasing height increased to the height of $40.0 \mathrm{~cm}$, the terminal velocity of the cylinder with fillet radius of $0 \mathrm{~mm}$ increased $70.9 \%$. In contrast, the terminal velocity of the cylinder with fillet radius of $4.0 \mathrm{~mm}$ increased $82.3 \%$. Although the hydrodynamic drag of the cylinder with fillet radius of $0 \mathrm{~mm}$ reduced significantly, the risingt rate of terminal velocity was lowest. The cavity reduced the hydrodynamic drag, while simultaneously increased the upward buoyancy force.

According to the paper from Thoroddsen's group (Sci. Adv. 2017, 3, e1701558), the underwater cavity can only be retained when the vehicle moves in the vertical direction, because the hydrostatic pressure gradient cannot support the cavity shape. The direction of buoyancy force is always opposite to the direction of cylinder movement. Furthermore, the increase of buoyancy force induced by the cavity was much larger than the hydrodynamic drag force. Thus, the buoyancy force can be seen as the additional drag force. In our experiment, we aim to develop a strategy to reduce the drag of the blunt cylinder. The outline shape of the cylinders was changed by the attached cavity, and the buoyancy force of attached cavity cann't be neglected. Therefore, we think that it is more reasonable to consider the influence of the 
buoyancy force on evaluating the underwater drag reduction effect of the attached cavity on the cylinder.

\section{S16. The effective density of the cavity attached hydrophilic cylinders with different releasing height and fillet radius}

We calculated the effective density of the cavity attached to cylinder by using the equation as follows.

$$
\rho_{e f f}=\frac{V_{s} \rho_{s}}{V_{S C}}
$$

where the $V_{S}$ and $\rho_{S}$ are the volume and density of the cylinder, respectively, and $V_{S C}$ is the volume of the cylinder-cavity. The effective densities of the cavity attached hydrophilic cylinders with different fillet radii and releasing heights were shown in the Figure S16. With the increasing of the releasing heights, the cavity shapes tended to be more streamlined, and the total volume was also increased. As a result, the effective density of the cylinder-cavity decreased sharply. In the case of the cylinder released from $40 \mathrm{~cm}$ height, the effective density was in the region of 1.4 to $1.9 \mathrm{~g} / \mathrm{cm}^{3}$. Such effective density was much lower than the density of the bare cylinder, but still a little larger than that of water. According to work from Thoroddsen's group (Soft matter 2019, 15, 6278-6287), the effective density of the sphere-cavity was remarkably close to that of water. We guess that there are two possible reasons caused such differences between the cylinders and sphere. The first possible one was that the geometric shapes influenced the stability of the underwater cavity and then the cavity shapes. The falling cylinder was probably much more unstable under water. The second possible one was that the releasing heights in our experiments were much smaller. Thus, there was not enough air entrapped by the cylinders to induce fully developed cavity. We will investigate the impacting behaviors of the cylinders releasing from much larger heights in the further study. The gaps of the effective density between the cavity attached to spheres and cylinders also indicated that the spheres provided much higher drag reduction effect. Therefore, our results indicates that the percentage of hydrodynamic drag in the upward resultant force is relatively 
low in case of the streamlined cavity, and the cavity can reduce the drag force effectively.

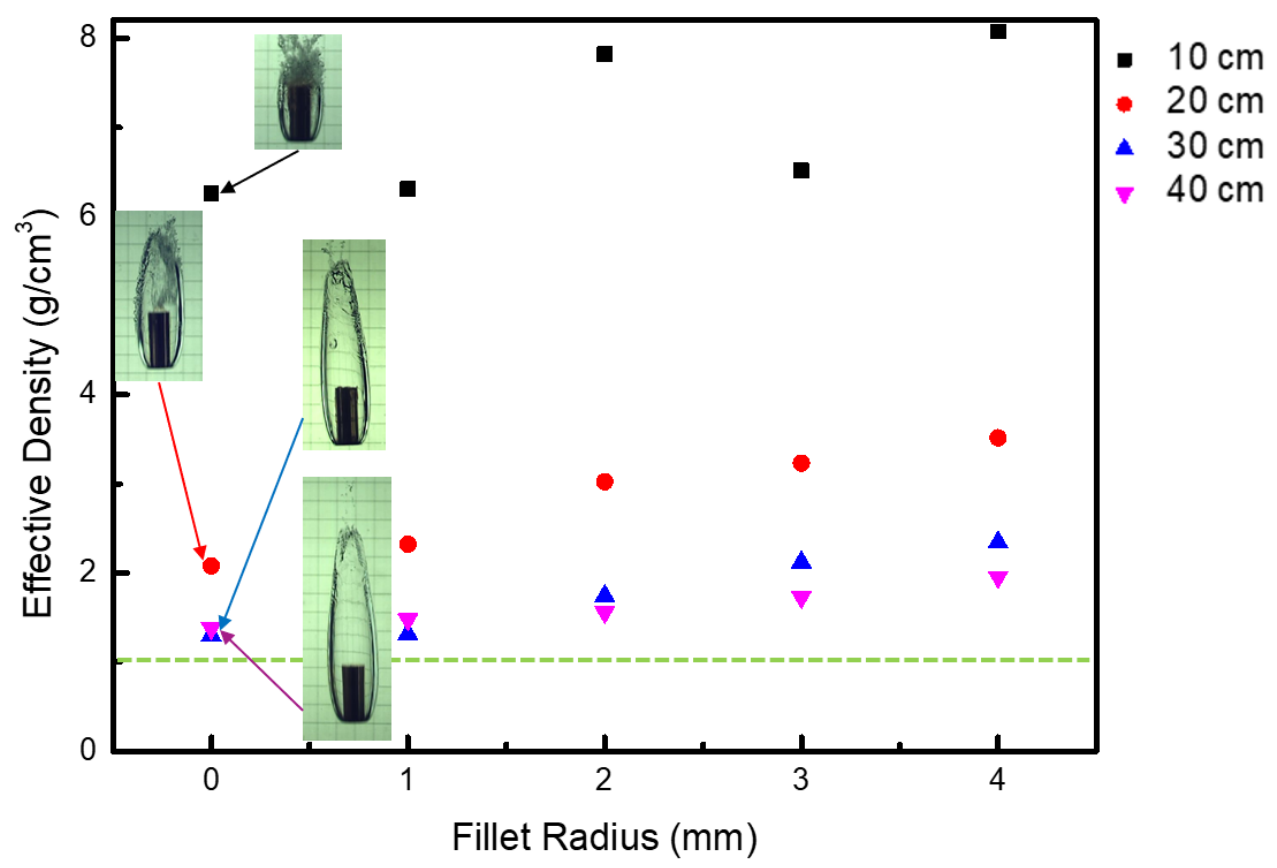

Figure S16 The effective densities of the cavity attached to hydrophilic cylinders with different fillet radii $(0,1.0,2.0,3.0$, and $4.0 \mathrm{~mm})$ and releasing heights $(10.0,20.0$, 30.0 , and $40.0 \mathrm{~cm})$.

S17. The calculation of the total volume of the cavity retaining cylinder 


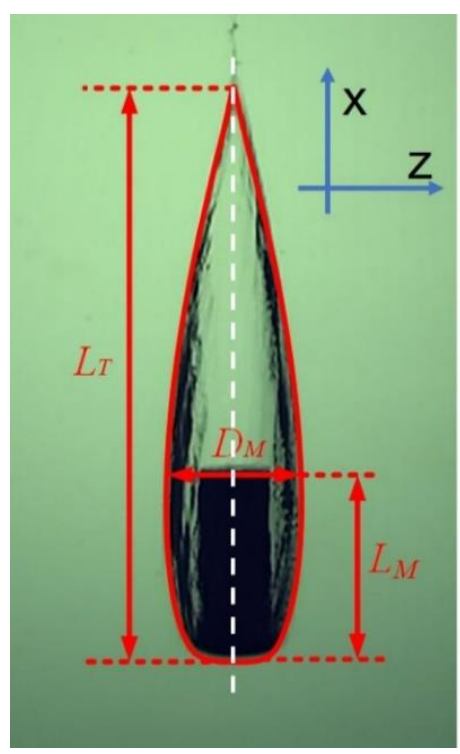

We calculated the total volume using the algebraic curve fitting method (Sci.

$A d v$. 2017, 3, e1701558). The outline of the cavity retaining cylinder was fitted by the algebraic curve, as shown in Figure 4a middle, then we calculated the integral of the algebraic equation in the cylindrical coordinates. First, the shape of the cavity retaining cylinder exhibited bilateral symmetry; thus, we only needed to fit the outline in one side. Second, the outlines of the cavities were divided into two pieces. In the vertical direction, from the cavity tail to the latitude with the maximal diameter $\mathrm{D}_{\mathrm{M}}$ on the cavities was considered as a parabola $\mathrm{z}_{1}$. From the latitude $\mathrm{D}_{\mathrm{M}}$ to the lower part of the cylinder was considered as another parabola $z_{2}$. The rear part of the cavity was fitted by the parabolic curve: $z_{1}=\frac{D_{M}}{2}\left[1-\left(\frac{x}{L_{T}-L_{M}}\right)^{2}\right]$. The lower part of the cavity was fitted by the parabolic curve: $z_{2}=x^{2}+\left[\frac{2 R-D_{M}}{2 L_{M}}-L_{M}\right] x+\frac{D_{M}}{2}$, where $\mathrm{R}$ is the diameter of the cylinder. The volume of the cavity was calculated using the integration method.

$\mathrm{V}=\int_{0}^{L_{T}-L_{M}} \pi z_{1}^{2} d x+\int_{0}^{L_{M}} \pi z_{2}^{2} d x$

Considering that the integration of $y_{2}$ is too complicated, the parabolic curve $y_{2}$ was approximated by the parabolic curve $y_{1}$, since the outline of the cavity was partly symmetrical from the tail to the latitude $\mathrm{D}_{\mathrm{M}}$. The volume function of the cavity can be written as:

$\mathrm{V}=\pi\left(\frac{D_{M}}{2}\right)^{2}\left(L_{M}+\frac{3 L_{M}{ }^{5}-10 L_{M}{ }^{3}\left(L_{T}-L_{M}\right)^{2}}{15\left(L_{T}-L_{M}\right)^{4}}+\frac{8\left(L_{T}-L_{M}\right)}{15}\right)$ 
Table S8. The calculated total volume $\left(\mathrm{cm}^{3}\right)$ of the cavity retaining hydrophilic cylinders with different releasing heights and fillet radii.

\begin{tabular}{cccccc}
\hline Radius $(\mathrm{mm})$ & & & & & \\
\hline 10.0 & & 1.0 & 2.0 & 3.0 & 4.0 \\
\hline 20.0 & 6.599 & 6.553 & 5.283 & 6.342 & 5.119 \\
\hline 30.0 & 19.876 & 17.795 & 13.661 & 12.789 & 11.749 \\
\hline 40.0 & 31.758 & 31.352 & 23.716 & 19.479 & 17.632 \\
\hline
\end{tabular}

Table S9. The calculated total volume $\left(\mathrm{cm}^{3}\right)$ of the cavity retaining hydrophobic cylinders with different releasing heights and fillet radii.

\begin{tabular}{cccccc}
\hline Radius $(\mathrm{mm})$ & 0 & 1.0 & 2.0 & 3.0 & 4.0 \\
\hline 10.0 & 8.021 & 8.433 & 6.464 & 7.838 & 5.444 \\
\hline 20.0 & 22.134 & 19.171 & 20.911 & 15.455 & 13.016 \\
\hline 30.0 & 31.723 & 32.159 & 25.425 & 22.330 & 18.823 \\
\hline 40.0 & 34.995 & 29.646 & 22.964 & 21.477 & 20.593 \\
\hline
\end{tabular}


Table S10. The calculated total volume $\left(\mathrm{cm}^{3}\right)$ of the cavity retaining superhydrophilic cylinders with different releasing heights and fillet radii.

\begin{tabular}{cccccc}
\hline Radius (mm) & & & & & \\
& 0 & 1.0 & 2.0 & 3.0 & 4.0 \\
\hline 10.0 & 6.758 & 8.230 & 6.196 & 9.257 & 7.304 \\
\hline 20.0 & 21.247 & 16.934 & 14.904 & 12.893 & 15.423 \\
\hline 30.0 & 31.461 & 25.918 & 21.107 & 17.630 & 15.581 \\
\hline 40.0 & 37.836 & 36.210 & 29.625 & 24.222 & 20.708 \\
\hline
\end{tabular}

Table S11. The calculated total volume $\left(\mathrm{cm}^{3}\right)$ of the cavity retaining superhydrophobic cylinders with different releasing heights and fillet radii.

\begin{tabular}{cccccc}
\hline Radius $(\mathrm{mm})$ & 0 & 1.0 & 2.0 & 3.0 & 4.0 \\
\hline 10.0 & 9.671 & 8.116 & 6.925 & 6.696 & 6.465 \\
\hline 20.0 & 21.932 & 21.654 & 20.027 & 19.371 & 17.154 \\
\hline 30.0 & 31.936 & 23.519 & 20.125 & 19.268 & 17.820 \\
\hline 40.0 & 39.251 & 34.834 & 27.889 & 26.534 & 24.539 \\
\hline
\end{tabular}

